Pacific Journal of Mathematics

INJECTIVE HULLS OF GROUP RINGS

Bander Brown and John William LaUren 


\title{
INJECTIVE HULLS OF GROUP RINGS
}

\author{
Kenneth A. Brown and John Lawrence
}

\begin{abstract}
We are concerned in this paper with the following question: When is the maximal right quotient ring of the group algebra $k G$ a right self-injective ring? In general, the maximal right quotient ring $Q(R)$ of a ring $R$ is a right $R$-submodule of the right injective hull $E(R)$ of $R$, and we may rephrase our question as: When does $Q(k G)=E(k G)$ ? Of course, a sufficient condition for this to occur is that $k G$ be right nonsingular, so that, for example, $E(k G)=Q(k G)$ when $k$ is a field of characteristic zero. However, $Q(k G)$ is often injective even when $k G$ is a singular ring; for example, when $G$ is finite, it is well-known that $k G$ is itself an injective ring.
\end{abstract}

Many of our results are concerned with the case of commutative group algebras. If $G$ is an abelian group and $k$ is a field of positive characteristic $p$, then $Z(k G)$, the singular ideal of $k G$, is the ideal generated by the augmentation ideal of the Sylow $p$-subgroup $H$ of $G$, [2], Corollary 3.5 and [11], Lemmas 3.1.6 and 8.1.8. We shall prove (Theorem 4.1), that if $H$ contains only finitely many elements of infinite height, then $Q(k G)$ is injective. Not all commutative group algebras have injective quotient rings, however; under the above hypothesis on $k$, we prove (Corollary 3.2) that if a group $T$ contains a subnormal Prüfer $p$-group, $Q(k T)$ is not selfinjective. Some commutative group algebras are not, of course, included among those covered by Theorem 4.1 and Corollary 3.2, so that even in the commutative case we are unable to offer a complete answer to our question.

Sections 3 and 4 are devoted, respectively, to the proofs of Corollary 3.2, which is in fact a slightly more general result than that quoted above, and Theorem 4.1. The rest of the paper is organized as follows. In $\S 2$ we obtain some technical results relating the injective hull of a group algebra to the injective hulls of certain subalgebras and quotient algebras; these results are used frequently in subsequent sections. In $\S 5$ we show that, although they fail in general to be injective, maximal quotient rings of commutative group algebras possess many of the well-known properties of injective rings. For example, if $Q$ is such a ring, $Z(Q)=J(Q)$, the Jacobson radical of $Q$, (Theorem 5.3), $Q / J(Q)$ is a regular self-injective ring, (Theorems 5.3 and 5.5), and idempotents may be lifted over $J(Q)$, (Proposition 5.4). In $\S 6$ we drop the assumption, common to $\S \S 4$ and 5 , that the group algebras under consideration are commutative, 
and prove a generalization of the fact that group algebras of finite groups are self-injective: If the right singular ideal of the group algebra $k G$ is nilpotent, then $Q(k G)$ is right self-injective. Finally, $\S 7$ contains a discussion of some open problems.

We would like to thank D. S. Passman for several suggestions and comments which have proved extremely helpful in the preparation of this paper.

2. Subgroups, quotient groups and subalgebras. In this section we assemble some preliminary results. We begin by listing some notation which will remain fixed through out the paper. If $R$ is a ring, $J(R)$ will denote the Jacobson radical of $R,(Z(R)$ will denote Jacobson radical of $R), Z(R)$ the right singular ideal of $R$, and $Q(R)$ the maximal right quotient ring of $R$. If it exists, we shall denote the classical right quotient ring of $R$ by $Q_{c l}(R)$. If $X$ is a subset of $R$, then $r-\operatorname{Ann}_{R}(X)$ and $l-\mathrm{Ann}_{R}(X)$ will denote repectively the right and left annihilators of $X$ in $R$, and we write $\operatorname{Ann}_{R}(X)$ (or simply $\mathrm{Ann}(X)$ ) for $r-\mathrm{Ann}_{R}(X) \cap l-\mathrm{Ann}_{R}(X)$. For an ideal $I$ of $R$, we write

$$
C_{R}(I)=\{\alpha \in R: \alpha \beta \in I, \beta \in R \Longrightarrow \beta \in I\} .
$$

We shall use the appropriate small German letter to denote the argumentation ideal of a group algebra; thus the augmentation ideal of $k G$ will be denoted by $g$. Finally, we shall sometimes denote the $p$-primary subgroup $G$ by $0_{p}(G)$.

We say that a subgroup $H$ of a group $G$ locally subnormal in $G$ if, for every finite subset $\left\{x_{1}, \cdots, x_{n}\right\}$ of $G, H$ is a subnormal subgroup of $\left\langle H, x_{1}, \cdots, x_{n}\right\rangle$. Note that if $H$ is an ascendent subgroup $G, H$ is locally subnormal in $G$.

Proposition 2.1. Let ke be a field and $G$ a group.

(i) If $H$ is a locally subnormal subgroup of $G$, and $Q(k G)$ is right self-injective, then $Q(k H)$ is right self-injective.

(ii) If $F$ is o subfield of $k$ and $Q(k G)$ is right self-injective, then $Q(F G)$ is right self-injective.

Proof. (i) Suppose the result is false, and let $E(k H)$ denote the injective hull of $k H_{\mid k H}$, By [7], §4.3, Propositions 1 and 3, since $Q(k H)$ is not self-injective there exists

$$
0 \neq \eta \in L=\operatorname{Hom}_{k H}(E(k H), E(k H))
$$

such that $\eta(k H)=0$.

Let $\left\{g_{i}: i \in I\right\}$ be a right transversal to $H$ in $G$, so that 


$$
k G_{\mid k H}=\sum_{i \in I}^{\oplus}\left(k H g_{i}\right)_{\mid k H} \subseteq \sum_{i \in I}^{\oplus}\left(E(k H) \bigotimes_{k H} g_{i}\right) .
$$

Now $T=\sum_{i \in I}^{\oplus}\left(E(k H) \otimes g_{i}\right)$ is a right $k G$-module, and in fact contains the submodule $k G_{\mid k G}$. Moreover $T_{\mid k G}$ is an essential extension of $k G_{\mid k G}$. This is easy to see if $H \triangleleft G$, since then $\left(E(k H) \otimes g_{i}\right)$ is a $k H$-module which is an essential extension of the $k H$-module $k H \otimes g_{i}$, for each $i \in I$. It follows by a simple inductive argument on the index of subnormality of $H$ in $G$, when $H$ is subnormal, and the proof may be completed in the present case by noting that if $T_{\mid k G}$ is not an essential extension of $k G_{\mid k G}$, there exists $0 \neq \alpha \in k G$ such that $T \cap \alpha k G=0$, so by puting $N=\langle H, \operatorname{supp} \alpha\rangle$ and noting that $H$ is subnormal in $N$ we may obtain a contradiction by considering the $k H$-submodule $(E(k H) \otimes k N)$ of $T$.

Since $Q(k G)_{\mid k G}$ is, by hypothesis, the injective hull of $k G_{\mid k G}$, there is an embedding of $T$ in $Q(k G)$. The $k H$-homomorphism $\eta$ can be extended by linearity to a $k G$-homomorphism $\bar{\eta}$ from $T$ into itself, and so, since $Q(k G)_{l k G}$ is injective $\bar{\eta}$ extends to a $k G$-homomorphism

$$
\bar{\eta}^{\prime}: Q(k G) \longrightarrow Q(k G) \text {. }
$$

Now since $\eta \neq 0, \bar{\eta}^{\prime} \neq 0$, but since $\bar{\eta}^{\prime}{ }_{1 k H}=\eta$, we have $\bar{\eta}^{\prime}(k G)=0$. By [7], $\S 4.3$, Propositions 1 and 3 , it follows that $Q(k G)$ is not selfinjective, a contradiction. Hence $Q(k H)$ is right self-injective.

(ii) This is proved by the obvious adaptation of the proof of (i).

It is not true that if $H \subseteq G$ and $Q(k G)$ is rightself-injective, then so is $Q(k H)$; see the example given after Corollary 3.2. There is however one case where the assumption that $H$ is locally subnormal may be removed:

LEMma 2.2. Let $H$ be a subgroup of finite index in the group $G$ and let $k$ be a field. Then $Q(k H)$ is right self-injective if and only if $Q(k G)$ is right self-injective.

Proof. Suppose first that $H$ is normal in $G$, and that $Q(k H)$ is right self-injective. Now $Q(k G)=k G \bigotimes_{k H} Q(k H)$, [9], Corollary 13, so we can adapt the proof of [12], Lemma 4 to show that $Q(k G)$ is right self-injective. In general, if we put $H^{0}=\bigcap_{g \in G} H^{g}$, so that $H^{0} \triangleleft G, H^{0} \subseteq H$, and $G / H^{0}$ is finite, then $Q(k H)$ right self-injective implies $Q\left(k H^{0}\right)$ right self-injective, by Proposition 2.1 (i), and the above observation applied to $Q(k G)=k G \otimes_{k H^{0}} Q\left(k H^{0}\right)$ shows that $Q(k G)$ is right self-injective.

Conversely, if $Q(k G)$ is right self-injective then so is $Q\left(k H^{0}\right)$, by Proposition 2.1 (i), and the above argument shows that $Q(k H)$ is right self-injective. 
It would be unreasonable to expect that one could obtain results relating the injectivity of a quotient ring of a group algebra involving a group $G$ to the injectivity of quotient rings of group algebras of images of $G$, and indeed, armed with the results of $\S 3$, the reader may very easily construct examples to show that there are no general results relating the structures of such quotient rings. There is however one case where such a result is available.

THEOREM 2.3. Let $k$ be a field of characteristic $p>0$, and let $H$ be a finite normal p-subgroup of the group $G$. If $Q(k(G / H))$ is right self-injective, then so is $Q(k G)$.

Proof. Put $C=C_{G}(H)$, so $G / C$ is finite and $C \cap H$ is a finite central $p$-subgroup of $C$. Since $C / C \cap H \cong C H / H, Q(k(C / C \cap H))$ is right self-injective by Lemma 2.2, while another application of the same lemma shows that $Q(k G)$ is right self-injective provided $Q(k C)$ is. Thus we may assume that $H$ is central in $G$, and moreover we may then assume that $H$ is cyclic, say $H=\langle x\rangle$. Assume that $o(x)=p$.

Since $k$ has characteristic $p, \mathfrak{h}$ is nilpotent, by [11], Lemma 3.1.6; (If $H=1$, there is of course nothing to prove.) Let $E$ denote the right $k G$-injective hull of $k G$, and for $0 \leqq i \leqq p$, put

$$
E_{i}=\left\{e \in E: e(x-1)^{i}=0\right\},
$$

so that

$$
0=E_{0} \leqq E_{1} \leqq \cdots \leqq E_{p}=E
$$

is a chain of $k G$-submodules of $E$, since $x$ is central in $G$.

We claim that $E_{1}$ is the right $k G / \mathfrak{k} G$-injective hull of $k G / \xi g$. First note that if we write $\hat{H}=\sum_{h \in H} h$, then $\hat{H} k G \subseteq E_{1}$, and, as $k G / \mathfrak{h} G$-modules,

$$
\hat{H} k G \cong k G / \hat{s} G,
$$

since $r-\operatorname{Ann}_{k G}(\hat{H})=\mathfrak{h} G$, by [11], Lemma 3.1.2. Moreover $\hat{H} k H$ is an eesential ideal of $k H$, being its unique minimal ideal, so that $\hat{H} k G$ is an essential right ideal of $k G$, by [2], Lemma 2.5, and it follows that $E_{1} \cap k G=\hat{H} k G$ is an essential $k G$-submodule of $E_{1}$, since $E$ is an essential extension of $k G_{\mid k G}$. Since $H k G$ is essential in $k G, E$ is the $k G$-injective hull of $\hat{H} k G$, so that $E_{1}$ contains a copy of the $k G / \mathfrak{h} G$-injective hull of $\hat{H} k G$. Thus since $E_{1}$ is an essential extension of $\hat{H} k G$, (as $k G / \mathfrak{h} G$-modules), our claim is proved.

By [7], §4.3, Propositions 1 and 3, the result will follow if we can show that if $h \in \operatorname{Hom}_{k G}(E, E)$ and $h(k G)=0$, Then $h=0$. Let 
$h \in \operatorname{Hom}_{k G}(E, E)$, then $h(k G)=0$. Clearly $h\left(E_{1}\right) \subseteq E_{1}$, and $h(\hat{H} k G)=0$. Since $k(G / H)$ is isomorphic to $k G / \mathfrak{h} G$, we deduce from [7], $\S 4.3$ Propositions 1 and 3 , the right self-injectivity of $Q(k(G / H))$, and the observations of the previous paragraph, that $h\left(E_{1}\right)=0$. Now for $1 \leqq i<p$ there is a $k G$-monomorphism from $E_{i+1} / E_{i}$ into $E_{1}$, given by multiplication by $(x-1)^{i}$ Suppose that we have shown for some $1 \leqq i<p$, that $h\left(E_{i}\right)=0$. We claim that this implies that $h\left(E_{i+1}\right)=0$. The map $h: E \rightarrow E$, annihilates $E_{i}$, so we have $h: E_{i+1} / E_{i} \rightarrow E_{1}$. Since $E_{1}$ is $k(G / H)$ injective, $h$ extends to a map $h^{\prime}: E_{1} \rightarrow E_{1}$ (since $E_{i+1} / E_{i} \subseteq$ $E_{1}$ by the above embedding). Let $\beta=(x-1)^{p-(i+1)} \in E_{i+1} / E_{i}$, then $h(\beta)=0$, hence $h^{\prime}(\hat{H} k(G / H))=0$, and so (by Lambek [7], §4.3, Prop. 1.3), $h^{\prime}\left(E_{1}\right)=0$. It follows that $h\left(E_{i}\right)=0$, as claimed.

Since $h\left(E_{1}\right)=0$, we deduce that $h=0$ and result is proved.

REMARK. It is clear that the argument used in the first paragraph of the above proof enables one to relax the hypothesis on $H$ to the extent that it need only be assumed that $H$ is a finite normal subgroup whose centre is a p-group. We do not know if Theorem 2.3 is true for an arbitrary finite normal subgroup $H$ of $G$, although it seems likely that this is the case.

For the reader's convenience, we end this section with a statement of the principal test for self-injectivity that we shall use in the succeeding two sections.

Proposition 2.4. A ring $R$ has a right self-injective maximal right quotient ring if and only if every $R$-homomorphism $f: I \rightarrow R$, where $I$ is a right ideal of $R$, can be extended to a homomorphism $f^{\prime}: J \rightarrow R$ where $J$ is an essential right ideal of $R$ with zero left annihilator.

For a proof of Proposition 2.4, see [14], Proposition XIV. 4.2.

3. Noninjective quotient rings. In this section we describe all those group algebras known to us whose maximal quotient ring fails to be self-injective.

THEOREM 3.1. Let $k$ be a field of characteristic $p>0$, and let $G=C_{p^{\infty}}$, the Pröfer p-group. Then $Q(k G)$ is not self-injective.

Proof. By Proposition 2.4, the result will follow if we can find an ideal $I$ of $k G$ with nonzero annihilator, and a homomorphism $f: I \rightarrow k G$ which has no extension to an ideal with zero annihilator. Let $C=\langle c\rangle$ be the subgroup of $G$ of order $p$, and put $I=\mathfrak{c g}$. For each 
$i \geqq 1$, let $H_{i}$ be the subgroup of $G$ of order $p^{i}$, let $\hat{H}_{i}=\sum_{h \in H_{i}} h$, and choose $\alpha_{i} \in k G$ such that

$$
(c-1) \alpha_{i}=\hat{H}_{i} .
$$

Note that this can certainly be done since $\hat{H}_{i} k H_{i}$ is the unique minimal ideal of $k H_{i}$.

Now define

$$
f: I \longrightarrow k G: \beta \longrightarrow \sum_{i=1}^{\infty} \alpha_{i} \beta \text {. }
$$

If $\beta=(c-1) \gamma$, say, where $\gamma \in \mathfrak{g} \cap k H_{n}$, some $n \geqq 1$, then

$$
(c-1) \alpha_{t} \gamma=0
$$

for all $t \geqq n$, so $f$ is a well-defined $k G$-homomorphism. We claim that $f$ has no proper extensions.

Notice first that if $\mathfrak{c g} \subsetneq J \triangleleft k G$, then $\mathrm{c} G \subseteq J$. For there exists $m \geqq 1$ such

$$
\mathfrak{c h}=\mathfrak{c g} \cap k H_{m} \subsetneq J \cap k H_{m},
$$

where $\mathfrak{h}$ is the augmentation ideal of $k H_{m}$, and since there is a unique chain of ideals in $k H_{m}$, we deduce that

$$
c H_{m} \leqq J \cap k H_{m} \text {. }
$$

Now if $\nu$ is any $k G$-homomorphism from $c G$ into $k G$, then since, by [11], Lemma 3.1.2, $c G$ is the annihilator in $k G$ of $\widehat{C}=\sum_{x \in C} x$, it is clear that

$$
\nu((c-1))=(c-1) \delta,
$$

for some $\delta \in k G$, and $\nu$ is simply multiplication by $\delta$. Thus if $f$ has a proper extension, there exists $\delta \in k G$ such that $f(x)=x \delta$ for all $x \in I$. Suppose that such an element $\delta$ exists, with $\delta \in k H_{s}$, say, and let $h$ be a generator of $H_{s+2}$. Then

$$
\begin{aligned}
f((c-1)(h-1))=\sum_{i=1}^{\infty} \hat{H}_{i}(h-1) & =\sum_{i=1}^{s+1} \hat{H}_{i}(h-1) \\
& =(c-1)(h-1) \delta,
\end{aligned}
$$

so that

$$
\left((c-1) \delta-\sum_{i=1}^{s+1} \hat{H}_{i}\right) \in \hat{H}_{s+2} k H_{s+2},
$$

since this ideal is the annihilator in $k H_{s+2}$ of $(h-1)$, by [11], Lemma 3.1.2. Note that since $(c-1) \delta \in k H_{s},(c-1) \delta-\sum_{i=1}^{s+1} H_{i}$ is a non- 
zero element of $k H_{s+1}$. However, it is clear that

$$
\hat{H}_{s+2} k H_{s+2} \cap k H_{s+1}=0 \text {, }
$$

so we have a contradiction.

Thus $f$ has no proper extensions, and since $\hat{C}$ annihilates $I$, the result follows.

COROLLARY 3.2. Let $k$ be a field of characteristic $p>0$, and let $G$ be a group containing a locally subnormal subgroup $H$, with $H \cong C_{p^{\infty}}$. Then $Q(k G)$ is not self-injective.

Proof. This is immediate from Theorem 3.1 and Proposition 2.1 (i).

Notes. (a) It is possible to strengthen Proposition 2.1, and with it Corollary 3.2 , by demanding only that, in the notation of [2], page $44,(H, G) \in \mathscr{P}$. However, since we are primarily interested in the case of group algebras of abelian groups, we shall not pursue this further.

(b) It is now a simple matter to construct an example to show that some hypothesis on the nature of the embedding of $H$ in $G$ in Proposition 2.1 is necessary. Thus if we fix a prime $p$, let $k$ be the field of $p$ elements and let $G=C_{\infty} 2 C_{\infty}$, where $C_{p^{\infty}}$ denotes infinite cyclic group, so that $G$ contains a subgroup $H$ with $H \cong C_{p \infty}$, then $k G$ is nonsingular by [2], Corollary 3.11 (i), so that $Q(k G)$ is right self-injective by [14], Proposition XII. 2.1, but Theorem 3.1 shows that $Q(k H)$ is not self-injective.

4. Injectivity of commutative quotient rings. Our aim in this section is to prove the following result.

THEOREM 4.1. Let $k$ be a field of characteristic $p>0$, and let $G$ be an abelian group with at most finitely many p-elements of infinite height. Then $Q(k G)$ is a self-injective ring.

We shall prove the above theorem by means of a sequence of lemmas. Note first that, by Proposition 2.1 (ii), we may assume without loss of generality that the coefficient field $k$ is algebraically closed. Accordingly, throughout $\S 4, p$ will denote a fixed prime and $k$ will be algebraically closed field of characteristic $p$.

LemmA 4.2. Let $B$ be a subgroup of finite index in the abelian group $G$, and suppose there exists a finite subgroup $H$ of $G$ such that 
$G=B H . \quad$ Let $I \triangleleft k G$, and suppose that the kG-homomorphism $f: I \rightarrow k G$ has no proper extensions. Then $I \cap k B \neq 0$.

Proof. Choose an ideal $L$ of $k H$ maximal with respect to the property that there exists $0 \neq \beta \in k B$ such that $L \beta \cong I$. Note that since $H$ is finite and $\{0\} \triangleleft k H$ has this property, there exists such an ideal $L$. If $L=k H$ then the lemma is already proved, so we may assume that $L \neq k H$. Let $L \beta k B=\sum_{v=1}^{t} \alpha_{v} k G$, and put

$$
T=\left\langle H, \operatorname{supp} \beta, f\left(\alpha_{v}\right): 1 \leqq v \leqq t\right\rangle .
$$

$T$ is a finitely generated group. Suppose $T \cap B=X \oplus Y$, where $X$ is finite and $Y$ is torsion-free. Then $T=\langle H, X\rangle \oplus Y$, where $\langle H, X\rangle$ is finite. Now $L \beta$ is a finite dimensional $k$-algebra, and by construction, $f(L \beta k T) \subseteq k T$. By [1], Theorem $\mathrm{B}, k T$ has a quasi-Frobenius quotient ring obtained by inverting the nonzero elements of $k Y$, so $f$ is induced on $L \beta k T$ by multiplication by $a c^{-1}$, where $a \in k T$ and $0 \neq c \in k Y$. Let $\gamma=\beta c$, so $0 \neq L \gamma \subseteq I$. Since, we have assumed that $L \neq k H$, we may choose an ideal $M$ (of $k H$ ) minimal over $L$. Then $M / L$ is an irreducible $k H$ module, hence $M=L+k \mu$ (1) and $N=$ ann $(M / L)$ has codim. 1 in $k H$. Thus $N+k=k H$ and $M N \subseteq L$ (2).

Now

$$
k G=(k H) B=(N+K) B=N k B+k B .
$$

By (1),

$$
M \gamma k B=L \gamma k B+\mu \gamma k B
$$

We claim that

$$
\mu \gamma k B \cap I=(0) .
$$

If not, suppose

$$
0 \neq \mu \gamma \gamma^{\prime} \in \mu \gamma k B \cap I
$$

Then

$$
M \gamma \gamma^{\prime}=L \gamma \gamma^{\prime}+k \mu \gamma \gamma^{\prime} \subseteq I
$$

and $0 \neq \mu \mu^{\prime} \in k B$, contradicting the maximality of $L$. Thus

$$
\begin{aligned}
M \gamma k G & =M \gamma(N k B+k B) \\
& =M N \gamma k B+M \gamma k B \\
& \cong L \gamma k B+(L \gamma k B+\mu \gamma k B) \\
& \cong L \gamma k B+\mu \gamma k B
\end{aligned}
$$

and we deduce from (5), that 
Since the map $f$ is induced on $L \gamma k B$ by multiplication by $a c^{-1}$, we may extend this map to

$$
f^{\prime}: M \gamma k G \longrightarrow k G
$$

by $x \rightarrow x a c^{-1} \in k G$, (by $\operatorname{def} \gamma$ ). Then we have a map

$$
f^{\prime \prime}: I+M \gamma k G \longrightarrow k G
$$

which extends $f$ (by (6) this is well-defined). By choice of $L, I+$ $M \gamma k G$ is strictly larger than $I$, a contradiction. This $L=k H$ and the proof is complete.

REMARK. We used the assumption that $k$ be algebraically closed in only one place in the above proof-namely, to ensure that a minimal ideal of $k H / L$ is a 1 -dimensional vector space over $k$. Thus we can drop this assumption if we know, for example, that $G$ is a $p$-group. We do not know whether it can be dropped in general.

LEMMA 4.3. (i ) Let $G$ be an abelian group whose torsion subgroup contains no elements of infinite height. If $H$ is a finite subgroup of $G$, there exists a finite subgroup $T$ of $G$, containing $H$, such that $T$ is a direct summand of $G$.

(ii) Let $P$ be an abelian p-group with only finitely many elements of infinite height, and let $H$ be the subgroup generated by those elements. Then $P / H$ contains no elements of infinite height.

Proof. (i ) It is clearly sufficient to assume that $H$ is a $p$ group and to find a $p$-group $T$ with the required properties. Now if $H$ is a $p$-group, we deduce, by successive applications of [11], Lemma 14.4.1 (iv), that there exists a finite pure subgroup $T$ of $0_{p}(G)$ such that $T$ contains $H$. Clearly $T$ is pure in $G$, and so by [4], Corollary 24.6, $T$ is a direct summand of $G$.

(ii) This is clear.

Proof of Theorem 4.1. We are given an abelian group $G$, a prime $p$ such that $G$ has only finitely many $p$-elements of infinite height, and a field $k$ of characteristic $p$, and we have to prove that $Q(k G)$ is injective.

As already remarked, we may assume that $k$ is algebraically closed. We begin by proving the result under the stronger assumption that $G$ has no $p$-elements of infinite height. In this case it follows that we may assume without loss that the torsion subgroup of $G$ contains no elements of infinite height. By Proposition 2.4, 
the result will follow if we can show that if $I$ is an ideal of $k G$ and $f \in \operatorname{Hom}_{k G}(I, k G)$ has no proper extensions, then $\operatorname{Ann}(I)=0$. Suppose that this is false, and let $f: I \rightarrow k G$ have no proper extensions, with Ann $(I) \neq 0$.

Let $W$ be a finitely generated subgroup of $G$ such that Ann $I \cap$ $k W \neq(0)$. Let $H$ be the torsion subgroup of $W$. If $H$ is a $p$-group, then as Ann $I \cap k H \neq 0$, we have $\hat{H} I=0$ (for $k H$ is a local ring with maximal ideal annihilated by $\hat{H}$ ). Choose $B$ a subgroup of finite index in $G$ such that $H \cap B=\langle 1\rangle$ and $H B=G$. Then

$$
I \cap k B \subset k k G \cap k B=(0)
$$

which contradicts Lemma 4.2 .

Now suppose $H=A \oplus C$, where $C$ is a $p^{\prime}$-group and $A$ is a p-group.

Let $1=\sum_{i=1}^{n} e_{i}$ be the decomposition of 1 in $k C$. Then $k G=$ $\sum_{i=1}^{n} e_{i} k G$ and $I=\sum_{i=1}^{n} e_{i} I$. Now $e_{i} k G$ is some twisted group algebra of $G / C$, but it is commutative and $k$ is algebraically closed, hence $e_{i} k G \cong k(G / C)$ [11, Lemma 1.2.9]. The map $f: I \rightarrow k G$ splits as $f=$ $\sum_{i=1}^{n} f_{i}$, where $f_{i}: e_{i} I \rightarrow e_{i} k G$ and since $f$ has no extension, each $f_{i}$ has no extension. Furthermore, since Ann $I \cap k W \neq 0$, we have $e_{j}(\operatorname{Ann} I \cap k W) \neq 0$ for some $j$. Thus $f_{j}: e_{j} I \rightarrow e_{j} k G$ does not extend and $\left(\mathrm{Ann}_{e j k G} e_{j} I\right) \cap e_{j} k W \neq 0$. But this is all happening in a ring isomorphic to $k(G / C)$ and under the isomorphism $e_{j} k W$ corresponds to $k(W / C)$ with $H / C$ a finite $p$-group. Our previous argument shows that this cannot occur.

Now allow $G$ to contain finitely many $p$-elements of infinite height, and let $N$ be the finite subgroup they generate. By Lemma 4.3 (ii), $G / N$ has no $p$-elements of infinite height, and so $Q(k(G / N))$ is self-injective by the first part of the proof. Hence, by Theorem $2.3, Q(k G)$ is self-injective, and the proof of Theorem 4.1 is complete.

5. The structure of commutative quotient rings. Throughout this section $G$ will denote an abelian group, $k$ a field of characteristic $p>0$, and $Q$ the maximal quotient ring of $k G$. Our main result is to demonstrate that $Q$ possesses many of the well-known properties of self-injective rings, as described in [5], pages 48-52, for example. In particular, we prove that $Q$ is continuous [15].

We begin by recalling that $Q$ is a commutative ring; in fact if we let $\mathscr{H}$ denote the ring of $k G$-homomorphism of the injective hull of $k G$ then $Q$ is isomorphic to the centre of $\mathscr{H},[7], \S 4.3$, Exercise 3. Our first objective is to prove Proposition 5.2, a result which is crucial to the proofs of the results which follow it. For its proof, we need the following technical lemma.

LEMMA 5.1. Let $R$ be the classical quotient ring of $k G$, and let 
$S=\left\{f_{i}: i \in I\right\}$ be a set of idempotents in $R$. Then $\operatorname{Ann}_{R}(S)$ is generated by the idempotents it contains.

Proof. Put $X=\operatorname{Ann}_{R}(S)=\beta\left(1-f_{i}\right) R$, and choose $x \in X$. There exists a finitely generated subgroup $T$ of $G$ such that $x \in R^{\prime}$, the classical quotient ring of $k T$. Note that $R^{\prime} \leqq R$, by [11], Lemma 1.1.4. Let $P$ be the Sylow $p$-subgroup of $T$, so $T=A \oplus P$, say, $P$ is finite, and

$$
R^{\prime}=Q_{c l}(k A) \bigotimes_{k} k P,
$$

since the right-hand side is an Artinian partial classical quotient ring of $k T$, and so is the whole quotient ring. Choose $e=e^{2} \in R^{\prime}$ such that $e x=x$, and the Goldie dimension of $e R^{\prime}$ is minimal for $e \in R^{\prime}$ with this property. Now $e \in Q \operatorname{ct}(k A)$; let $e=e_{1}+\cdots+e_{t}$ be the decomposition of $e$ as a sum of primitive idempotents of $Q_{c l}(k A)$, so that $0 \neq e_{j} x \in X$, for $1 \leqq j \leqq t$. The proof will be complete if we can show that $e_{j} \in X$ for $1 \leqq j \leqq t$. Let $e_{j} Q_{c l}(k A)=K_{j}$, an extension field of $k$, so that $e_{j} x \in e_{j} K_{j} P$, say $e_{j} x=e_{j} w_{j}$ for some $0 \neq w_{j} \in K_{j} P$, for $1 \leqq j \leqq t$. Fix $j \in\{1, \cdots, t\}$, and let $f \in S$. There exists a finitely generated subgroup $B$ of $G$ such that $A \subseteq B$, $0_{p}(B)=1$, and $f \in Q_{c l}(k B)$. Now $B \oplus P \subseteq G$, and

$$
R^{\prime \prime}=Q_{c l}(k(B \oplus P))=Q_{c l}(k B) \bigotimes_{k} k P,
$$

so $e_{j} R^{\prime \prime}=Q_{c l}(k B) e_{j} K_{j} P$. Since $e_{j} x \neq 0$, this implies that if $e_{j}=$ $\tau_{1}+\cdots+\tau_{m}$ is the decomposition of $e_{j}$ as a sum of primitive idempotents in $Q_{c l}(k B)$, then $\tau_{l} w_{j} \neq 0$ for $l=1, \cdots, m$. Since

$$
\begin{aligned}
& 0 \neq \tau_{l} w_{j} \in \bigcap_{i}\left(1-f_{i}\right) R, \\
& \tau_{l} w_{j}(1-f)=\tau_{l} w_{j},
\end{aligned}
$$

for $1 \leqq l \leqq m$.

Since $\tau_{l}$ is primitive in $Q_{c l}(k B)$, either $\tau_{l}(1-f)=0$ or $\tau_{l}(1-f)=\tau_{l}$, and (6) excludes the former possibility. Thus $e_{j}=$ $e_{j}(1-f) \in(1-f) R$, for $j=1, \cdots, t$, and since $f$ was an arbitrary element of $S$, the result is proved.

Proposition 5.2. An essential closure in $Q$ of an ideal $I$ of $Q$ is a direct summand of $Q$, and so is unique.

Proof. The ideal $(I \cap k G) Q$ is essential in $I$, so it is clearly sufficient to prove the result under the additional assumption that $I=(I \cap k G) Q$. Given any finite set $T_{\lambda}=\left\{x_{1}, \cdots, x_{n(\lambda)}\right\}$ in $G$, form the classical quotient ring $R_{\lambda}$ of $k\left\langle T_{\lambda}\right\rangle$, so $R_{\lambda}$ is a quasi-Frobenius ring, by [1], Theorem B. Note that $R_{\lambda}$ is a subring of $Q$, by Corollary 
13 of [9]. Choose an idempotent $f_{\lambda} \in R_{\lambda}$ such that $I \cap f_{\lambda} Q=0$, and the Goldie dimension of $f_{\lambda} R$ is maximal among idempotents of $R_{\lambda}$ with this property. Note that $f_{\lambda}$ is uniquely determined by these specifications. Index the set of all finite subsets of $G$ by $\wedge$, and put $J=\sum_{\lambda \in \wedge} f_{\lambda} Q$.

Now put $X=\operatorname{ann}(J)=\bigcap_{\lambda}(1-f) Q$; we claim that $W=X \oplus J$ is a dense ideal of $Q$. Put $R=\left\langle R_{\lambda}: \lambda \in \wedge\right\rangle$, a subring of $Q$ containing $k G$. Our claim will follow if we show that $W^{\prime}=(X \cap R) \oplus$ $(J \cap R)$ is a dense ideal of $R$, since $Q$ is an essential extension of $R$ as $R$-modules. Since $f_{\lambda} \in R$ for all $\lambda \in \wedge$, it is clear that $\operatorname{Ann}_{R}\left(W^{\prime}\right)^{2}=0$. However, by Lemma 5.1, $X \cap R=\operatorname{Ann}_{R}\left(\sum_{\lambda} f_{\lambda} R\right)$ is generated by the idempotents it contains, so by a second application of Lemma 5.1, $\operatorname{Ann}_{R}\left(W^{\prime}\right)=0$, thus proving our claim.

It follows from [7], page 98, that the projection map $X \oplus$ $J \rightarrow J$ is afforded by multiplication by an element $e \in Q$. Since $\left(e-e^{2}\right)(X \oplus J)=0$, and $X \oplus J$ is dense, $e$ is an idempotent of $Q$. Note that $J \subseteq e Q$, while $X \cap e Q=0$. Since $I \subseteq X, I \cap e Q=0$, we claim that $I$ is essential in $(1-e) Q$. Suppose that this is false, and choose $0 \neq \alpha \in k G \cap(1-e) Q$ such that $\alpha Q \cap I=0$. Put $A=\langle\operatorname{supp} \alpha\rangle$, and let $U=Q_{c l}(k A)$, so that, since $U$ is a quasi-Frobenius ring, [1]. Theorem $\mathrm{B}$, there exists a nonzero idempotent $f \in U$ such that $\alpha U$ is an essential $U$-submodule of $f U$. Since $U \subseteq Q$, [9], $f \in Q$. We prove that $\alpha Q$ is essential in $f Q$. Note first that $f U=(f U \cap k A) U$, and $\alpha k A$ must necessarily be essential in $f U \cap k A$. It follows as in the proof of [2], Lemmas 2.2 and 2.5, that $\alpha k G$ is essential in $(f U \cap k A) k G$, and so, since $Q_{\mid k G}$ is an essential extension of $k G_{\mid k G}$, it is easy to check that $\alpha Q$ is essential in $(f U \cap k A) Q=f Q$. Since $I \cap \alpha Q=0$, we must have $I \cap f Q=0$. It follows that, if $A=T_{\mu}$, $(\mu \in \wedge)$, then $f=f_{\mu} f$; that is, $f \in J$, and so $f \in e Q$. However, since $\alpha \in(1-e) Q$ and $\alpha Q$ is essential in $f Q, f \in(1-e) Q$. Hence $f \in$ $(1-e) Q \cap e Q$, so $f=0$, a contradiction. Therefore $I$ is essential in $(1-e) Q$, as climed, and the proof is complete.

Recall that a ring $R$ is left continuous if it satisfies the two conditions:

1. For any left ideal $I$ there is an idempotent $e$ such that $\operatorname{Re}$ is an essential extension of $I$.

2. If $\operatorname{Re}, e=e^{2}$, is isomorphic to a left ideal $I$, then $I$ is generated by an idempotent.

\section{THEOREM 5.3. $Q$ is continuous.}

Proof. We have proved in Proposition 5.2, that $Q$ satisfies condition 1 (above). Since $Q$ is commutative and rationally complete, condition 2 is automatic, indeed, under the hypothesis of condition $2, I=e R$. 
Proposition 5.4. $Z(Q)=J(Q), Q / Z(Q)$ is a regular ring and idempotents may be lifted over $Z(Q)$.

Proof. This follows from the fact that $Q$ is continuous [15].

REMARK. The proof of the main result of [3], which related the singular ideals of certain group algebras to the singular ideals of the group algebras of subgroups of finite index, is heavily dependent on the fact that, in the maximal right quotient rings of the group algebras under consideration, the right singular ideal conincides with the Jacobson radical. It was this proof which provided the initial motivation to study the injective hulls of group algebras, as we wished to examine the possibility of extending the results of [3]. Since we now know that not all quotient rings of group algebras are self-injective, it might seem at first sight that there is no possibility of using the methods of [3] in a more general setting; however Theorem 5.3 shows that such an extension has not yet been completely ruled out.

THEOREM 5.5. Let $H$ be the Sylow p-subgroup of $G$. Then $Q / Z(Q)$ is isomorphic to the maximal quotient ring of $k(G / H)$. In particular, $Q / Z(Q)$ is a self-injective ring.

Proof. We split the argument into two steps.

(i ) $Q / Z(Q)$ is self-injective.

The proof of (i) closely follows the proof of the corresponding result for selt-injective rings; see for example [5], Theorem 2.21. We first show that if $e$ and $f$ are idempotents in $Q$ such that $T=$ $e Q \cap f Q \neq 0$, then $\bar{e} \bar{Q} \cap \bar{f} \bar{Q} \neq 0$, where $\bar{Q}=Q / Z(Q)$. Choose an ideal $K$ of $Q$ such that $T \cap K=0, T+K$ is essential in $Q$, and $K$ is maximal among ideals with these properties. Clearly $K$ is essentially closed in $Q$, so that $K$ is direct summand of $Q$ by Proposition 5.2. Let $N$ be a maximal essential extension of $T$ in $e Q$; then $N$ is essentially closed in $Q$, so, again using Proposition 5.2, $N$ is a direct summand of $Q$, and so of $e Q$. Thus $N=\tau Q$, say, with $\tau=\tau^{2}$, $\tau e=\tau$, and $N \cap K=0$. Repeating this argument, we also obtain an element $h=h^{2} \in Q$ such that $h Q$ is a maximal essential extension of $T$ in $f Q$, and $h K=0$. Then $(\tau-h)$ is annihilated by $T+K$, an essential ideal of $Q$, and so $(\tau-h) \in Z(Q)$. However, $\bar{\tau} \neq 0$, and $\bar{\tau} \in \bar{e} \bar{Q} \cap \bar{f} \bar{Q}$, since $\bar{\tau}=\bar{h}$.

Now let $I$ be an ideal of $\bar{Q}$, and choose a set $\left\{\bar{e}_{i}\right\}$ of orthogonal idempotents in $\bar{Q}$, maximal with respect to containment in $I$. By Theorem 5.3, $X=\sum_{i} \bar{e}_{i} \bar{Q}$ is essential in $I$, and it follows from the fact that $\bar{Q}$ is nonsingular, (Theorem 5.3), that $\bar{Q}$-homomorphisms 
from $I$ to $\bar{Q}$ are determined by their action on $X$. By Proposition 5.4 we can lift $\left\{\bar{e}_{i}\right\}$ to a set $\left\{e_{i}\right\}$ of idempotents of $Q$. Let $f$ be a $\bar{Q}$-homomorphism from $I$ to $\bar{Q}$, and put $f\left(\bar{e}_{i}\right)=\bar{x}_{i} \in \bar{Q}$. By the arguments of the first paragraph, $W=\sum_{i} e_{i} Q$ is the direct sum of the ideals $e_{i} Q$. There exists an idempotent $e$ of $Q$ such that $W$ is essential in $e Q$, by Proposition 5.2. We claim that $U=(1-e) Q+W$ is a dense ideal of $Q$. For $Z=\operatorname{Ann}_{Q}(U) \subseteq e Q$, so it $Z \neq 0, Z \cap$ $W \neq 0$, since $W$ is essential in $e Q$. Since $W$ is generated by the idempotents it contains, this is clearly impossible. It follows that the map

$$
U \longrightarrow Q:\left\{\begin{aligned}
(1-e) \gamma & \longrightarrow 0 \quad \forall \gamma \in Q \\
e_{i} & \longrightarrow e_{i} x_{i} \quad \forall i
\end{aligned}\right.
$$

is afforded by multiplication by an element $q$ of $Q$. Thus $\bar{q} \bar{e}_{i}=\bar{x}_{i}$, for all $i$, and so $f: I \rightarrow \bar{Q}$ extends to a $\bar{Q}$-endomorphism of $\bar{Q}$. By Baer's criterion, [7], page 88, (i) follows.

(ii) Let $R=k G+Z(Q) / Z(Q)$. We shall show that $(Q / Z(Q))_{\mid R}$ is an essential extension of $R_{\mid R}$.

Note that

$$
\begin{aligned}
R=k G+Z(Q) / Z(Q) & \cong_{\text {ring }} k G / k G \cap Z(Q) \\
& =k G / Z(k G)=k G / \mathfrak{b} G \\
& \cong k(G / H),
\end{aligned}
$$

by [2], Corollary 3.5, so that (i) and (ii) together imply Theorem 5.5. This is because $R$ is nonsingular, [2], Corollary 3.5, so essential ideals of $R$ are dense, and therefore (ii) implies that $Q / Z(Q)$ is certainly a partial quotient ring of $R$, by [5], Chapter $2 \mathrm{C}$, so that

$$
Q(R)=Q(Q / Z(Q))=Q / Z(Q),
$$

by (i) and [7], §4.3, Propositions 2 and 3.

We proceed to prove (ii). Let $\alpha \in Q \backslash Z(Q)$, let $D=\{d \in k G$ : $\alpha d \in k G\}$, a dense ideal of $k G,[14]$, Lemma IX. 1.5, and define

$$
L=\{\beta \in k G: \exists A \subseteq H, A \text { finite, such that } \alpha \beta \hat{A} \in k G\},
$$

where $\hat{A}=\sum_{a \in A} a$. Note that if $A_{1}$ and $A_{2}$ are finite $p$-subgroups of $G$, then, putting $B=\left\langle A_{1}, A_{2}\right\rangle, \hat{B}$ is a $k G$-multiple of both $\hat{A}_{1}$ and $\hat{A}_{2}$, since $\hat{B} k B$ is the unique minimal ideal of $k B$. It is thus easy to see that $L$ is an ideal of $k G$. Now $L \supseteq \mathfrak{h} G$; we claim that $L / \mathfrak{h} G$ is an essential ideal of $k G / \mathfrak{h} G$.

Let $\gamma \in k G \backslash \mathfrak{h} G$. Since $0_{p}(\langle\operatorname{supp} \gamma\rangle)$ is a summand of $\langle\operatorname{supp} \gamma\rangle$, it is clear that there exists $\phi \in k\langle\operatorname{supp} \gamma\rangle$ such that $0_{p}(\langle\operatorname{supp} \phi\rangle)=1$ and $\gamma-\dot{\phi} \in \mathfrak{b} G$. Moreover there exists $\eta \in k\langle\operatorname{supp} \phi\rangle$ such that $\phi \eta=e \xi$, 
where $e$ is a primitive idempotent of $k\langle\operatorname{supp} \phi\rangle$ and $\xi \in C_{k G e}(0)$. Since our aim is to prove that $\gamma k G \cap L \supsetneq \mathfrak{h} G$, we can assume that $\gamma=e \xi$. Since $D$ is dense, there exists $d \in D$ such that $d \gamma \neq 0$; we choose $d$ so that $d=d e$. Let $d=\sum_{i=1}^{n} d_{i} g_{i}$, where $d_{i} \in k H$ and $\left\{g_{i}\right\}$ forms part of a transversal to $H$ in $G$. Note that if we put $F=\langle\operatorname{supp} d$, supp $\gamma\rangle$, then we can choose the elements $g_{i} \in F$ so that $H \cap\left\langle g_{i}\right.$ : $1 \leqq i \leqq n\rangle=1$ and $\operatorname{supp} \gamma \leqq\left\{g_{i}: 1 \leqq i \leqq n\right\}$. We assume that the set $\left\{g_{i}\right\}$ has been chosen in this way. There exists a finite subgroup $A$ of $H$ such that $d_{i} \in k A$ for all $i=1, \cdots, n$. Since $\hat{A} k A$ is essential in $k A$, there exists $\mu \in k A$ such that

$$
0 \neq \mu d=\sum_{i=1}^{m} \hat{A} \lambda_{i} g_{i},
$$

where $0 \neq \lambda_{i} \in k, \quad 1 \leqq i \leqq m$, and $1 \leqq m \leqq n$. Put $\omega=\sum_{i=1}^{m} \lambda_{i} g_{i}$. Now

$$
\gamma \mu d=e \xi \mu d=\xi \mu d \neq 0,
$$

since $0 \neq \mu d \in k G e$ and $\xi \in C_{k G_{e}}(0)$. It follows that $\gamma \omega \neq 0$. However $\hat{A} \omega \in D$, so that

$$
\alpha \gamma \omega \hat{A} \in k G,
$$

and so $\gamma \omega \in L$ by definition of $L$. Let $F=0_{p}(F) \oplus F^{\prime}$, choosing $F^{\prime}$ so that $\gamma \in k\langle\operatorname{supp} \gamma\rangle \leqq k F^{\prime}$, and $\omega \in k\left\langle g_{i}: 1 \leqq i \leqq n\right\rangle$, a subalgebra of $k F^{\prime}$, so that $\gamma \omega \notin \mathfrak{h} G$ since $k F^{\prime} \cap \mathfrak{h} G=0$. Therefore $L / \mathfrak{h} G$ is an essential ideal of $k G / \mathfrak{h} G$.

If $x \in L$, there exists a finite $p$-subgroup $Y$ of $G$ such that $\alpha x \hat{Y} \in(k G \cap \hat{Y} Q)$. Since $k G \cap \hat{Y} Q$ is the annihilator in $k G$ of $\mathfrak{b}$, it follows from [11], Lemma 3.1.2 that $k G \cap \hat{Y} Q=\hat{Y} k G$. Thus $\alpha x \hat{Y}=\hat{Y} \delta$, say, where $\delta \in k G$, and so $(\alpha x-\delta) \in \operatorname{Ann}_{Q}(\hat{Y})$. Now $\hat{Y} k Y$ is an essential ideal of $k Y$, being its unique minimal ideal, so $\hat{Y} k G$ is an essential ideal of $k G$, as in [2], Lemmas 2.2 or 2.5. Thus $\hat{Y} Q$ is essential in $Q$, and so $\alpha x-\delta \in Z(Q)$. Hence $\alpha x \in k G+Z(Q)$; so $\alpha L \subseteq$ $k G+Z(Q)$, and the proof of (ii) will be completed by showing that $\alpha L \nsubseteq Z(Q)$.

To prove that $\alpha L \not \subset Z(Q)$, we first show that we may assume that $\alpha$ is an idempotent. For if we put $V=\{\alpha \in Q: \exists L \triangleleft k G, L / \mathfrak{h} G$ essential in $k G / \mathfrak{h} G$, such that $\alpha L \subseteq Z(Q)\}$, then clearly $V \triangleleft Q$, and if $V \supsetneq Z(Q)$ then by Theorem 5.3 and Proposition 5.4, $\mathrm{V}$ contains a nonzero idempotent of $Q$. Thus we assume henceforth that $\alpha$ is a nonzero idempotent in $V$, and aim for a contradiction.

Let $S=Q_{c l}(k G)$; by [1], Theorem B, every finite set of elements of $S$ is contained in a quasi-Frobenius subring, and straightforward local arguments show that $Z(S)=\mathfrak{h} S$ and that

$$
S / Z(S) \cong Q_{c l}(k G / \mathfrak{h} G) \cong Q_{c l}(k(G / H)) .
$$


By [2], $k(G / H)$ is nonsingular, so another elementary local argument shows that $S / Z(S)$ is a regular ring. Now since $S$ is a subring of $Q, Z(S) \subseteq S \cap Z(Q)$, and in fact we have equality here, since if $S \cap Z(Q) \supsetneq Z(S), S \cap Z(Q) / Z(S)$ would contain a nonzero idempotent of $S / Z(S)$, and since $Z(S)=\mathfrak{h} S$ is nil, this would imply that $S \cap Z(Q)$ contained a nonzero idempotent, a contradiction. Hence

$$
S / Z(S)=S / \mathfrak{h} S=S / S \cap Z(Q) \cong S+Z(Q) / Z(Q),
$$

and

$$
k G / \mathfrak{h} G=k G / \mathfrak{h} S \cap k G \cong k G+\mathfrak{h} S / \mathfrak{h} S \cong Q_{c l}(k G+\mathfrak{h} S / \mathfrak{h} S)=S / \mathfrak{h} S .
$$

Thus since $L=\{t \in k G: \alpha t \in Z(Q)\}$ has the property that $L / \mathfrak{G} G$ is essential in $k G / 5 G$, (9) implies that $L S / \mathfrak{k} S$ is an essential ideal of $S / \mathfrak{b} S$, so that, by (8), $L S+Z(Q) / Z(Q)$ is an essential of $S+Z(Q) / Z(Q)$. As we have already observed, $S / Z(S)$ is regular and idempotents may be lifted over $Z(S)$, so $L S / Z(S)$ contains an ideal $J / Z(S)$ which is essential in $L S / Z(S)$, and so in $S / Z(S)$, and such that $J=\mathfrak{b} S+J^{\prime}$, where $J^{\prime}$ is generated by idempotents of $S$.

We claim that $J^{\prime}$ is an essential ideal of $S$. Let $0 \neq \beta \in k G$. We split the proof that $\beta S \cap J^{\prime} \neq 0$ into two cases. First, suppose that $\beta \notin \mathfrak{h} G$; then there exist elements $\psi \in \mathfrak{h} S$ and $\mu \in S \backslash \mathfrak{h} S$ such that $\beta \mu \notin \mathfrak{h} S$ but $\beta \mu+\psi \in J^{\prime}$, since $J / \mathfrak{h} S$ is essential in $S / \mathfrak{h} S$. Let $\psi=a c^{-1}$, where $a, c \in k G$ and $a \in \mathfrak{h} G$. It follows that there exists a finite $p$ subgroup $M$ of $G$ such that $\widehat{M} a=0$, and so

$$
\widehat{M}(\beta \mu+\psi)=\hat{M} \beta \mu \in J^{\prime} \cap \beta S .
$$

But $M \beta \mu \neq 0$ since $0 \neq \beta \mu \notin \mathfrak{m} S=\operatorname{Ann}_{S}(\hat{M})$, by [11], Lemma 3.1.2. Suppose on the other hand that $\beta \in \mathfrak{h} G$. Let $T_{0}=\langle\operatorname{supp} \beta\rangle$, put $U=0_{p}\left(T_{0}\right)$, so there exists $W \cong T_{0}$ such that $T_{0}=U \oplus W$, and write $\beta=\sum_{j=1}^{w} \beta_{j} g_{j}$, where $\beta_{j} \in \mathfrak{t}, 1 \leqq j \leqq w$, and $\left\{g_{j}: 1 \leqq j \leqq w\right\}$ are distinct elements of $W$. There exists an element $x \in k U$ such that $0 \neq x \beta=\hat{U}\left(\sum_{j} \mu_{j} g_{j}\right)$, where $\mu_{j} \in k$ and at least one $\mu_{j} \neq 0,1 \leqq j \leqq w$. Put $0 \neq \nu=\sum_{j} \mu_{j} g_{j} \in k W$. Since $Q_{c l}(k W)$ is a regular subring of $S$, there exists $\bar{\delta} \in S$ such that $0 \neq \nu \bar{\delta}=e^{\prime}$ is an idempotent in $S$, and $0 \neq \hat{U} e^{\prime} \in \beta S$. Now there exists $\rho \in S$ and $\bar{\omega} \in \mathfrak{h} S$ such that $e^{\prime} \rho \notin \mathfrak{h} S$ and $e \rho+\bar{\omega} \in J^{\prime}$. Since $\operatorname{Ann}_{S}(\hat{U}) \subseteq \mathfrak{h} S$, as in the previous case, $U e^{\prime} \rho \neq 0$, so that

$$
0 \neq \hat{U} e^{\prime}\left(e^{\prime} \rho+\bar{\omega}\right)=\hat{U} e^{\prime} \rho+\hat{U} e^{\prime} \bar{\omega} \in J^{\prime} \cap \beta S,
$$

as required. Since $S=Q_{c l}(k G), J^{\prime}$ is an essential ideal of $S$, as claimed.

Since $Q$ is the maximal quotient ring of $S$ it follows that $J^{\prime} Q$ is an essential ideal of $Q$. Now $Q$ is commutative, so the product 
of two idempotents of $Q$ is an idempotent, and so, recalling that $\alpha$ is an idempotent and that $J^{\prime}$ is generated by idempotents, $\alpha J^{\prime} \in Z(Q)$ if and only if $\alpha J^{\prime}=0$. However if $\alpha J^{\prime}=0$, then since $J^{\prime} Q$ is essential we deduce that $\alpha \in Z(Q)$, a contradiction. Hence $\alpha J^{\prime} \nsubseteq Z(Q)$, so $\alpha L \nsubseteq Z(Q)$, and the proof of (ii) is complete.

For the last result of $\S 5$ we impose a restriction on the abelian groups under consideration.

Proposition 5.6. The Goldie dimension of $Q$ is finite if and only if $A$, the direct sum of the Sylow $q$-subgroups of $G$ for $q \neq p$, is finite. If $A$ is finite, then

$$
Q=Q_{1} \oplus \cdots \oplus Q_{n},
$$

where $n$ is the number of distinct irreducible $k A$-modules, $Q_{i}$ is a uniform local ring, and $J\left(Q_{i}\right)=Z\left(Q_{i}\right)$ is nil, for $1 \leqq i \leqq n$. If we write $Q_{i} / J\left(Q_{i}\right)=K_{i}$, then

$$
K_{i} \cong K \bigotimes_{k} k_{i},
$$

where, if $H$ is the Sylow p-subgroup of $G, K$ is the quotient field of $k(G / H \oplus A)$, and

$$
k A \cong k_{1} \oplus \cdots \oplus k_{n}
$$

is the decomposition of $k A$ as a direct sum of fields.

Proof. If $A$ is infinite, then $k A$ is regular, since every finite set of elements of $k A$ is contained in a semisimple Artinian subring, but $k A$ is not Artinian, [11], Theorem 10.1.1, so $k A$ has infinite Goldie dimension. Since $k G$ is a free $k A$-module, $k G$ must then have infinite Goldie dimension, and so therefore must $Q$. Suppose conversely that $k A$ has Goldie dimension $n<\infty$, and note first that $k H$ is uniform, since if $0 \neq \alpha, \beta \in k H$, then putting $B=\langle\operatorname{supp} \alpha$, $\operatorname{supp} \beta\rangle, k B$ has a unique minimal ideal. Since $k(H \oplus A)$ is isomorphic as a ring to $\sum_{i=1}^{n}{ }^{\oplus} k_{i} H$, where $k_{i}$ is an extension field of $k$, it follows that $k(H \oplus A)$ has Goldie dimension $n$, so $k G$ has dimension $n$ by [1], Lemma 2.5. Therefore $Q$ has Goldie dimension $n$.

Suppose now that $A$ is finite, so by [4], Corollary 24.6, $G=$ $A \oplus C$, where the torsion subgroup of $C$ is a $p$-group, and

$$
k G \cong k A \otimes_{k} k C=\sum_{i=1}^{n}{ }^{\oplus} k_{i} C,
$$

where

$$
k A \cong k_{1} \oplus \cdots \oplus k_{n}
$$


is the decomposition of $k A$ as a direct sum of fields, so $k_{i}$ is an extension field of $k$. Thus

$$
Q(k G)=\oplus \sum_{i=1}^{n} Q\left(k_{i} C\right),
$$

by [7], $\S 4.3$, Proposition 9 , and $Q_{i}=Q\left(k_{i} C\right)$ is a uniform $k_{i} C$-module, and so a priori a uniform ring. It follows from Theorem 5.3 and Proposition 5.4 that $Q_{i}$ is local, and that $Z\left(Q_{i}\right)=J\left(Q_{i}\right)$.

We next show that $J\left(Q_{i}\right)$ is nil. Let $q \in J\left(Q_{i}\right)$, and represent $q$ as an element of $\operatorname{Hom}_{k_{i} C}\left(D, k_{i} C\right)$, where $D$ is a dense ideal of $k_{i} C$, as in [7], §4.3. As noted in the proof of Theorem 5.3, $I=\operatorname{ker} q \cap$ $D \neq 0$, where $\operatorname{ker} q$ denotes the kernel of $q$ as an element of $\operatorname{Hom}_{k_{i} c}(E, E)$, where $E$ is the injective hull of $k_{i} C$. (Notice that since $k_{i} C$ is commutative, $Q_{i}$ is the centre of $\operatorname{Hom}_{k_{i} c}(E, E)$, [7], $\S 4.3$, Exercise 3.) Since $0 \neq q Q_{i} \cap k_{i} C \subseteq \operatorname{Ann}_{k_{i} c}(I)$, the proof of Theorem 4 shows that there exists of finite subgroup $T$ of $C$ such that $I \subseteq \mathrm{t} k_{i} C$. We claim that we may assume that $I \subseteq k_{i} T \neq 0$. For as in the proof of Theorem $4,0 \neq \hat{Y} \gamma \in I$, for some regular element $\gamma$ of $k_{i} C$ and a finite $p$-subgroup $Y$ of $C$. Since $\gamma$ is a unit of $Q_{i}$, it follows that $Y \in I$, so replacing $T$ by $\langle T, Y\rangle$ gives the desired conclusion.

Let $L=\operatorname{Ann}_{k_{i} C}(I)$, and note that since $I \cap k T \neq 0, L \cong \mathrm{t} k_{i} C$, by [11], Lemma 3.1.2. We claim that for all $n \geqq 1 q^{n} D \subseteq t^{n} k_{i} C$. Since $q D \subseteq L$, this is certainly true when $n=1$. Suppose it is known to be true for $n=m-1$, some $m>1$, and let $\beta \in q^{m} D$, say $\beta=q \delta$, where $\delta \in{ }^{m-1} k_{i} C$. Let $X=\langle\operatorname{supp} \delta, \operatorname{supp} \beta, Y\rangle$, so $X$ is a finitely generated abelian group and by [1], Theorem B there exists an element $a c^{-1}$ of $Q_{c l}(k X)$ such that $q \delta=\delta a c^{-1}$ and $q \hat{Y}=\hat{Y} a c^{-1}=0$, since $\hat{Y} \cong I$. It follows that $a \in \operatorname{Ann}_{k_{i} C}(\hat{Y})=\mathfrak{y} k_{i} C \cong t_{k_{i}} C$, so that

$$
\beta=q \delta=\delta a c^{-1} \cong 1^{m} Q_{i} \cap k_{i} C=\mathrm{t}^{m} k_{i} C,
$$

as claimed. (The last equality holds since $\mathrm{t}^{m} k_{i} C$ is an annibilator ideal of $k_{i} C$.) Since $T$ is a finite $p$-subgroup of $C$, it follows from Lemma 3.1.6 of [11] that $\mathrm{t}_{i} C$ is nilpotent, say $\mathrm{t}^{r} k_{i} C=0$, so that $q^{r} D=0$. Since $D$ is a dense ideal of $k_{i} C$ and $Q_{i}$ is an essential extension of $k_{i} C_{\mid k_{i} c}$, it follows that $\operatorname{Ann}_{Q_{i}}(D)=0$, so that $q^{r}=0$. Thus $J\left(Q_{i}\right)$ is nil.

Finally, since $Q_{i}=Q\left(k_{i} C\right)$, the last part of the proposition follows from Theorem 5.5 and the fact that $Q\left(k_{i} C\right)=k_{i} \bigotimes_{k} Q(k C)$, [9], Theorem 11.

REMARK. If, instead of assuming that $0_{p^{\prime}}(G)$ is finite, we assume that $0_{p}(G)$ is finite, then it can be shown that the Jacobson radical of $Q$ is nilpotent. The proof is similar to that of [3], Theorem 2.2. 
We do not know if $Z(Q)$ is nil in general. This is certainly not the case if we drop the hypothesis that $G$ is an abelian group, since it is then no longer true even that $Z(k G)$ is nil; see, for example, [2], Example 4.4.

6. Injectivity of noncommutative quotient rings. We can offer no conjecture as to how Theorem 4.1 might generalize to group algebras of non-abelian groups. In this section we outline what little is known in the non-abelian setting. We begin by recalling that a sufficient condition for $Q(k G)$ to be right self-injective is that the group algebra $k G$ be right nonsingular, and that the results of [2], [3], and [13] provide many examples of such group algebras. Note that by [2], Lemma 3.1, $N(k G)$, the sum of the nilpotent ideals of $k G$, is always contained in $Z(k G)$, the sum of the nilpotent ideals of $k G$, is always contained in $Z(k G)$. The main result of this section that if $N(k G)$ and $Z(k G)$ are "not too big", $Q(k G)$ is right selfinjective.

THEOREM 6.1. If $k G$ is a group algebra such that $Z(k G)$ is nilpotent, then $Q(k G)$ is right self-injective.

Proof. Let $p$ be the characteristic of $k$. By [2], Lemma 3.1, $N(k G)$ is nilpotent, so that in the notation of [11], Chapter $8, \S 1$ $\Delta^{p}(G)$ is finite, by [11], Theorem 8.1.12. Put $C=C_{G}\left(\Delta^{p}(G)\right)$, so that $C \triangleleft G$ and $G / C$ is finite. Since $Z(k C) \subseteq Z(k G)$, by [2], Lemma 2.5, $Z(k C)$ must also be nilpotent. Now $T=\Delta^{p}(C) \subseteq C \cap \Delta^{p}(G)$, and so $T$ is a finite normal $p$-subgroup of $C$. Thus by [11], Theorem 8.1.0 and Lemma 3.1.6,

$$
\mathrm{t} C=N(k C)=Z(k C) .
$$

It follows from Theorem 3.16 of [2] that $k(C / T)$ is right nonsingular, so that by [5], Corollary 2.31, $Q(k(C / T))$ is right selfinjective. We now deduce from Theorem 2.3 that $Q(k C)$ is right self-injective, and so by Lemma $2.2 Q(k G)$ is right self-injective.

Notes. (i) Necessary and sufficient conditions for the hypothesis of Theorem 6.1 to hold, expressed in terms of the nonsingularity of certain group algebras, are given in Theorem 3.2 of [3]. If one restricts attention to a class of group algebras for which Conjecture A of [2], page 48, is known to be true, that is a class of group algebras for which $Z(k G)$ is known to equal $J(k L(G)) k G$, where $L(G)$ is the unique maximal locally finite normal subgroup of $G$, then 'one can obtain conditions expressed in terms of the struc- 
ture of $k L(G)$, using [11], Corollary 8.1.14. We leave the details to the interested reader.

(ii) An obvious question arises when one considers possible generalizations of Theorems 4.1 and 6.1: if $k$ is a field and $G$ and $H$ are groups such that $Q(k G)$ and $Q(k H)$ are right self-injective, is $Q(k(G \times H))$ right self-injective? We have been unable to answer this question, but it may be worth mentioning the following special case. Let $k$ have characteristic $p>0$, and suppose $G$ is an abelian group with only finitely many $p$-elements of infinite height, and $H$ is such that $k H$ has an Artinian classical right quotient ring. Then $Q(k(G \times H))$ is right self-injective. The proof involves a straightforward, though tedious, reduction to the case where $Q_{c l}(k H)$ is a division ring, and then an adaptation of the arguments of $\S 4$. Notice that the case $G=1$ is covered by Theorem 6.1, by [14], Lemma II. 2.5.

7. Open problems. The most obvious problem arising from the foregoing results is that of removing the gap between the results of $\S \S 3$ and 4 . Specifically, we have been unable to answer:

(i) Which group algebras of abelian groups have injective quotient rings?

Perhaps the simplest group algebra for which our methods do not provide answer is the following:

Let $A=\left\langle x_{1}: i=1,2, \cdots, x_{1}^{p}=1, x_{i}^{p^{i-1}}=x_{1},\left[x_{i}, x_{j}\right]=1\right\rangle$, where $p$ is a prime, and let $k$ be a field of characteristic $p$. It is easy to see that $A$ has $(p-1)$ nonidentity elements of infinite height, so $Q(k A)$ is self-injective by Theorem 4.1. Now let $G$ be the direct product of infinitely many copies of $A$. We ask as a special case of (i):

(ii) Is $Q(k G)$ self-injective?

About the results of $\S \S 3.4$, and 5, one may ask the blanket question:

(iii) How do the results of $\S \S 3-5$ generalize to noncommutative group algebras?

By analogy with the results of [2] and [3], one might hope for a positive answer to the following question. Let $L(G)$ denote the unique maximal locally finite normal subgroup of $G$, (see [2]).

(iv) If $Q(k L(G))$ is right self-injective, is $Q(k G)$ right selfinjective?

To illustrate one difficulty associated with (iv), consider the following special case. Let $k$ be the field of $p$ elements, and let $G=C_{p}<C_{\infty}$, the Wreath product of a cyclic group of order $p$ by an infinite cycle. Thus $; G$ has an elementary abelian normal subgroup $A$, and $G / A \cong C_{\infty}$. By [9], Corollary 12, 


$$
Q(k G) \supseteqq Q(k A) \bigotimes_{k A} k G \supseteqq k G,
$$

and so, by Theorem 4.1 applied to $k A, Q(k G)$ may be viewed as the maximal right quotient ring of a twisted polynomial rings over a self-injective ring. However, such quotient rings are not in general self-injective; for an example, see [8].

Finally, as explained in Section 5, we are particularly interested in the following question arising from Theorem 5.3, in view of its bearing on the results of [3].

(v) If $Q$ is the maximal right quotient ring of a group algebra, is $J(Q)=Z(Q)$ ?

\section{REFERENCES}

1. K. A. Brown, Artinian quotient rings of group rings, J. Algebra, 49 (1977), 63-80.

2. - The singular ideals of group rings, Quart. J. Math., (2), 28 (1977), 41-60.

3. - The singular ideals of group rings II, to appear.

4. L. Fuchs, Abelian Groups, (Pergamon), 1960.

5. K. Goodearl, Ring Theory: Nonsingular Rings and Modules, (Marcel Dekker), 1976.

6. C. R. Hajarnavis, On Small's theorem, J. London Math. Soc., (2), 5 (1972), 596-600.

7. J. Lambek, Lectures on Rings and Modules, (Ginn-Blaisdell), 1966.

8. J. Lawrence, Maximal quotient rings of twisted polynomial rings, to appear.

9. K. Louden, Maximal quotient rings of ring extensions, Pacific J. Math., 62 (1976), 489-496.

10. W. May, Invariants for commutative group algebras, Illinois J. Math., 15 (1971), 525-531.

11. D.S. Passman, The Algebraic Structure of Group Rings, (Interscience), 1977.

12. - On the ring of quotients of a group ring, Proc. Amer. Math. Soc., 33 (1972), 221-225.

13. R. L. Snider, On the singular ideal of a group algebra, Comm. in Algebra, 4 (1976), 1087-1089.

14. B. Stenstrom, Rings of Quotients, (Springer-Verlag), Berlin, 1975.

15. Y. Utumi, On continuous rings and self injective rings, Trans. Amer. Math. Soc., 118 (1965), 158-173.

Received April 14, 1978 and in revised form February 26, 1979. Some of the research for this paper was carried out while the authors were attending the 1977 Summer Research Institute of the Canadian Mathematical Congress at McGill University, Montreal. The first author was supported financially by the British Science Research Council, and the second author was partially supported by a University of Waterloo Research Grant 131-7052.

UNIVERSITY OF WARWICK

COVENTRY CV4 7AL

UNITED KINGDOM

AND

UNIVERSITY OF WATERLOO

WATERLOO, ONTARIO

CANADA 



\section{PACIFIC JOURNAL OF MATHEMATICS}

\section{EDITORS}

DONALD BABBITT (Managing Editor)

University of California

Los Angeles, CA 90024

HUgo RossI

University of Utah

Salt Lake City, UT 84112

C. C. Moore and ANDrew OGG

University of California

Berkeley, CA 94720

\section{J. DUGUNDJI}

Department of Mathematics

University of Southern California

Los Angeles, CA 90007

R. FinN and J. MILGRaM

Stanford University

Stanford, CA 94305

\section{ASSOCIATE EDITORS}
E. F. BECKENBACH
B. H. NeumanN
F. WOLF
K. YOSHIDA

\section{SUPPORTING INSTITUTIONS}

UNIVERSITY OF BRITISH COLUMBIA CALIFORNIA INSTITUTE OF TECHNOLOGY

UNIVERSITY OF CALIFORNIA

MONTANA STATE UNIVERSITY

UNIVERSITY OF NEVADA, RENO

NEW MEXICO STATE UNIVERSITY

OREGON STATE UNIVERSITY

UNIVERSITY OF OREGON
UNIVERSITY OF SOUTHERN CALIFORNIA

STANFORD UNIVERSITY

UNIVERSITY OF HAWAII

UNIVERSITY OF TOKYO

UNIVERSITY OF UTAH

WASHINGTON STATE UNIVERSITY

UNIVERSITY OF WASHINGTON

The Supporting Institutions listed above contribute to the cost of publication of this Journal, but they are not owners or publishers and have no responsibility for its content or policies.

Mathematical papers intended for publication in the Pacific Journal of Mathematics should be in typed form or offset-reproduced, (not dittoed), double spaced with large margins. Please do not use built up fractions in the text of the manuscript. However, you may use them in the displayed equations. Underline Greek letters in red, German in green, and script in blue. The first paragraph or two must be capable of being used separately as a synopsis of the entire paper. Please propose a heading for the odd numbered pages of less than 35 characters. Manuscripts, in triplicate, may be sent to any one of the editors. Please classify according to the scheme of Math. Reviews, Index to Vol. 39. Supply name and address of author to whom proofs should be sent. All other communications should be addressed to the managing editor, or Elaine Barth, University of California, Los Angeles, California, 90024.

50 reprints to each author are provided free for each article, only if page charges have been substantially paid. Additional copies may be obtained at cost in multiples of 50 .

The Pacific Journal of Mathematics is issued monthly as of January 1966. Regular subscription rate: $\$ 84.00$ a year (6 Vols., 12 issues). Special rate: $\$ 42.00$ a year to individual members of supporting institutions.

Subscriptions, orders for numbers issued in the last three calendar years, and changes of address should be sent to Pacific Journal of Mathematics, P.O. Box 969, Carmel Valley, CA 93924, U.S.A. Older back numbers obtainable from Kraus Periodicals Co., Route 100, Millwood, NY 10546.

PUBLISHED BY PACIFIC JOURNAL OF MATHEMATICS, A NON-PROFIT CORPORATION

Printed at Kokusai Bunken Insatsusha (International Academic Printing Co., Ltd.). 8-8, 3-chome, Takadanobaba, Shinjuku-ku, Tokyo 160, Japan.

Copyright (C) 1979 by Pacific Journal of Mathematics Manufactured and first issued in Japan 


\section{Pacific Journal of Mathematics}

\section{Vol. 85, No. $2 \quad$ October, 1979}

Charles A. Akemann and Steve Wright, Compact and weakly compact derivations of $C^{*}$-algebras ........................... 253

Dwight Richard Bean, Andrzej Ehrenfeucht and George Frank McNulty, Avoidable patterns in strings of symbols ................... 261

Richard Clark Brown, Notes on generalized boundary value problems in Banach spaces. I. Adjoint and extension theory.................

Kenneth Alexander Brown and John William Lawrence, Injective hulls of group rings .................................... 323

Jacob Burbea, The Schwarzian derivative and the Poincaré metric ....... 345

Stefan Andrus Burr, On the completeness of sequences of perturbed polynomial values ....................................

Peter H. Chang, On the characterizations of the breakdown points of quasilinear wave equations..............................

Joseph Nicholas Fadyn, The projectivity of $\operatorname{Ext}(T, A)$ as a module over

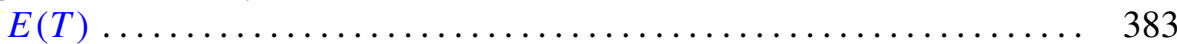

Donald Eugene Maurer, Arithmetic properties of the idèle discriminant .... 393

Stuart Rankin, Clive Reis and Gabriel Thierrin, Right subdirectly irreducible semigroups ................................. 403

David Lee Rector, Homotopy theory of rigid profinite spaces. I ........ 413 Raymond Moos Redheffer and Wolfgang V. Walter, Comparison theorems for parabolic functional inequalities................

H. M. (Hari Mohan) Srivastava, Some generalizations of Carlitz's theorem

James Alan Wood, Unbounded multipliers on commutative Banach algebras....

T. Yoshimoto, Vector-valued ergodic theorems for operators satisfying norm

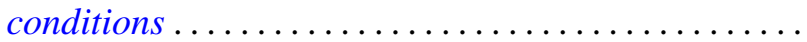

Jerry Searcy and B. Andreas Troesch, Correction to: "A cyclic inequality and a related eigenvalue problem".

Leslie Wilson, Corrections to: "Nonopenness of the set of Thom-Boardman maps" 